\title{
BARRERAS PARA LA DETECCIÓN OPORTUNA DEL CÁNCER CERVICOUTERINO EN COLOMBIA: UNA REVISIÓN NARRATIVA
}

Isabel Garcés Palacio ${ }^{1}$, Anderson Rocha Buelvas ${ }^{2}$

\section{Resumen}

Objetivo. Identificar las barreras socio-culturales, psicológicas, del sistema de salud y de acceso que impiden el diagnóstico oportuno del cáncer cervical en mujeres Colombianas según la literatura existente.

Métodos. Una revisión entre enero de 2003 y junio de 2014. La búsqueda se realizó en las fuentes de información: Pubmed, LILACS, The Cochrane Library, Cancer Library, CANCERMondial, Ministerio de Salud y Protección Social y Repositorios.

Resultados. De 40 publicaciones, se incluyeron 22 resultados de investigación en Colombia. Finalmente se incluyeron 10 estudios correspondientes a barreras socioculturales, 1 a barreras psicológicas, 12 barreras de los sistemas de salud y acceso.

Conclusiones. Se encontraron pocos estudios que demuestran la existencia de barreras relacionadas con la oportunidad de decisión y acción, de acceso a la atención y de calidad de la atención del cáncer cervicouterino en Colombia.

Palabras clave: neoplasias uterinas, accesibilidad a los servicios de salud, detección precoz del cáncer, salud de la mujer, países en desarrollo, características culturales (DeCS).

\footnotetext{
${ }^{1}$ Grupo de Investigación en Epidemiología. Facultad Nacional de Salud Pública. Universidad de Antioquia.

${ }^{2}$ Grupo de Investigación de Salud Pública y Desarrollo Social, Maestría en Salud Pública y Desarrollo Social. Fundación Universitaria del Área Andina.
} 


\title{
BARRIERS TO EARLY DETECTION OF CERVICAL CANCER IN COLOMBIA: A NARRATIVE REVIEW
}

\author{
Isabel Garcés Palacio, Anderson Rocha Buelvas
}

\section{Abstract}

Objective. To identify socio-cultural, psychological, health system and accessibility barriers that prevents early diagnosis of cervical cancer in Colombian women according to existing literature.

Methods. An update between January 2003 and June 2014. The search was conducted on the information sources: PubMed, LILACS, Science Direct, Cochrane Library, Cancer Library, CANCERMondial, Ministry of Health, Welfare, and Repositories.

Results. Of 40 publications, 22 Colombian research results were included. Finally, 10 studies concerning socio-cultural barriers were included, 1 to psychological barriers, 12 to barriers of health and access systems.

Conclusions. Few studies that demonstrated the existence of barriers related to the opportunity to make decisions and take actions, access to health care and quality of health care for cervical cancer in Colombia were found. 


\section{CONHECIMENTO, ATITUDES E PRÁTICAS DOS ESTUDANTES ESCOLARES PARA PREVENIR A DENGUE: UMA REVISÃO NARRATIVA}

Isabel Garcés Palacio, Anderson Rocha Buelvas

\section{Resumo}

Objetivo. Identificar as barreiras socioculturais, psicológicas, do sistema de saúde e de acesso que evitam o diagnóstico precoce de câncer cervical em mulheres colombianas de acordo com a literatura existente.

Metodologia. Uma revisão entre janeiro de 2003 e junho de 2014. A pesquisa foi realizada nas seguintes fontes de informação: Pubmed, LILACS, The Cochrane Library, Cancer Library, CANCERMondial, Ministério da Saúde e Proteção Social e repositórios.

Resultados. De 40 publicações, 22 resultados da investigação na Colômbia foram incluídos. Finalmente, foram incluídos 10 estudos para barreiras socioculturais, 1 para barreiras psicológicas, 12 barreiras dos sistemas de saúde e de acesso.

Conclusões. Encontraram-se poucos estudos que demonstram a existência de barreiras relacionadas com a oportunidade de decisão e ação, de acesso aos cuidados e da qualidade dos cuidados do câncer cérvico-uterino na Colômbia. 


\section{Introducción}

El cáncer cervico-uterino es una enfermedad prevenible y tratable no obstante, cerca de 528.000 mujeres son diagnosticadas con este tipo de cáncer, anualmente en el mundo, de las cuales poco más de la mitad mueren (1). Esta situación, representa un problema de salud pública en los países de bajos y medianos ingresos, que ocasionan importantes pérdidas económicas (2,3). La mortalidad del cáncer femenino se comporta de manera similar (8/100.000 para cáncer cervico-uterino y 10.8/100.000 para cáncer de seno) (3). En los últimos treinta años, la incidencia de cáncer de cuello uterino en Colombia ha disminuido, sin embargo, es actualmente el segundo tipo de cáncer más común en las mujeres. En América Latina y el Caribe es siete veces más alta la mortalidad por esta enfermedad que en los Estados Unidos $(4,5)$, y solo el $9 \%$ de los años potencialmente perdidos por esta enfermedad ocurren en países desarrollados(6).

Las consecuencias producidas por esta enfermedad afectan, especialmente, los ingresos económicos de las mujeres y el cuidado de menores de edad o familiares mayores a su cargo. La afectación en la calidad de vida de esta población altera la economía alrededor del mundo $(3,6)$. Los gobiernos, los sistemas de salud y los individuos invierten grandes cantidades de recursos en el diagnóstico y tratamiento de esta enfermedad potencialmente prevenible (2), siendo más alta en países en vía de desarrollo (7), donde además cuentan los costos

indirectos producidos por el ausentismo laboral y el transporte, entre otros (8).
En el mundo la citología cervico-uterina ha sido el eje central de los programas de detección temprana del cáncer cervico-uterino, logrando reducir sustancialmente la mortalidad en Europa por la implementación de programas organizados de tamizaje (9). No obstante, cerca de tres cuartas partes de las mujeres que en Colombia han accedido a una citología durante los últimos tres años, no han accedido a un seguimiento (10), ya que muchas no regresaron por sus resultados o estos no les fueron entregados (11). Esta situación no es ajena para Colombia ni para los programas de prevención implementados en la región de Las Américas, los cuales no han mejorado la situación; contrariamente, se espera que el número anual de casos diagnosticados aumente de 68.000 casos en 2008 a 126.000 en 2025 (12) con una manifestación diferencial, desde luego al interior de los países y para el caso colombiano, dadas las diferencias económicas, sociales y la situación de salud entre las regiones y subregiones (13). Es por ello que esta revisión pretende explorar la literatura existente de las diferentes barreras de acceso a la atención oportuna de esta enfermedad.

\section{Materiales y métodos}

Se realizó una revisión bibliográfica de las publicaciones científicas que abordan el tema de las barreras que impiden el diagnóstico oportuno del cáncer cervicouterino en Colombia encontrándose, a partir de la selección y evaluación de documentos, que las barreras estudiadas en Colombia son las socioculturales, las psicológicas y las del sistema de salud y de acceso propiamente dichas. 
Para la búsqueda de artículos científicos publicados entre enero de 2003 y junio de 2014 se consultaron varias fuentes de información: bases de datos Pubmed y LILACS; la red global independiente The Cochrane Library y la Cancer Library de (National Cancer Institute of National Institutes of Health) de los Estados Unidos; el sitio web CANCERMondial que contiene las bases de datos GLOBOCAN, Cancer Incidence in Five Continents (CI5) y World Health Organisation (WHO) manejada por la Sección de Vigilancia del Cáncer de la International Agency for Research on Cancer (IARC), la Biblioteca del Ministerio de Salud y Protección Social y Tesis de Repositorios de la Universidad Nacional de Colombia y Universidad de Antioquia en Tabla 1.

Tabla 1. Registro de revisión de datos.

\begin{tabular}{|c|c|c|c|c|c|c|c|c|}
\hline \multicolumn{2}{|c|}{$\begin{array}{l}\text { Nombre de la } \\
\text { Base de Datos }\end{array}$} & PubMed & LILACS & $\begin{array}{l}\text { The } \\
\text { Cochrane } \\
\text { Library }\end{array}$ & $\begin{array}{c}\text { Cancer } \\
\text { Library } \\
\text { (NA- } \\
\text { TIONAL } \\
\text { CANCER } \\
\text { INSTITU- } \\
\text { TE) }\end{array}$ & $\begin{array}{l}\text { CANCER- } \\
\text { Mondial } \\
\text { GLOBO- } \\
\text { CAN } \\
\text { CI5 } \\
\text { WHO }\end{array}$ & $\begin{array}{l}\text { Ministerio } \\
\text { Salud y } \\
\text { Protección } \\
\text { Social }\end{array}$ & $\begin{array}{l}\text { Reposito- } \\
\text { rios }\end{array}$ \\
\hline \multicolumn{9}{|c|}{ Años consultados: 2003-2014 } \\
\hline \multicolumn{2}{|c|}{$\begin{array}{l}\text { Términos de } \\
\text { búsqueda }\end{array}$} & $\begin{array}{l}\text { MeSH } \\
\text { Cervix } \\
\text { neoplasm } \\
\text { Cancer } \\
\text { screening/ } \\
\text { Early } \\
\text { detection } \\
\text { of cancer } \\
\text { Accessibi- } \\
\text { lity, } \\
\text { Health } \\
\text { Services } \\
\text { Libre } \\
\text { Diagnosis } \\
\text { delay } \\
\text { Treatment } \\
\text { delay }\end{array}$ & $\begin{array}{l}\text { Decs } \\
\text { Cáncer } \\
\text { del cuello } \\
\text { uterino, } \\
\text { Prevención } \\
\text { del cáncer } \\
\text { de cuello } \\
\text { uterino } \\
\text { Acceso } \\
\text { a los } \\
\text { servicios de } \\
\text { salud, } \\
\text { Detección } \\
\text { precoz del } \\
\text { cáncer, } \\
\text { Libre } \\
\text { Diagnóstico } \\
\text { tardío } \\
\text { Tratamiento } \\
\text { tardío }\end{array}$ & $\begin{array}{l}\text { MeSH } \\
\text { Cervix } \\
\text { neoplasm } \\
\text { Cancer } \\
\text { screening/ } \\
\text { Early } \\
\text { detection } \\
\text { of cancer } \\
\text { Accessibi- } \\
\text { lity, } \\
\text { Health } \\
\text { Services } \\
\text { Libre } \\
\text { Diagnosis } \\
\text { delay } \\
\text { Treatment } \\
\text { delay }\end{array}$ & $\begin{array}{l}\text { Libre } \\
\text { Cérvix } \\
\text { cáncer, } \\
\text { protocol, } \\
\text { diagnosis }\end{array}$ & $\begin{array}{l}\text { Libre } \\
\text { Mortality, } \\
\text { incidence, } \\
\text { cervix } \\
\text { cancer, } \\
\text { prevention, } \\
\text { handbook }\end{array}$ & $\begin{array}{l}\text { Libre } \\
\text { Diagnóstico } \\
\text { tardío } \\
\text { Tratamiento } \\
\text { tardío }\end{array}$ & $\begin{array}{l}\text { Libre } \\
\text { Diagnóstico } \\
\text { tardío } \\
\text { Tratamiento } \\
\text { tardío }\end{array}$ \\
\hline \multirow{5}{*}{$\begin{array}{l}\text { RESUL- } \\
\text { TADOS }\end{array}$} & $\begin{array}{l}\text { Idioma } \\
\text { (s) }\end{array}$ & Inglés & Castellano & Inglés & Inglés & Inglés & Castellano & Castellano \\
\hline & $\begin{array}{l}\text { Cualquier } \\
\text { campo. }\end{array}$ & 62.414 & 1.217 & 1.883 & 56 & 14 & 8 & 100 \\
\hline & $\begin{array}{l}\text { Materia } \\
\text { (Major } \\
\text { Topic) }\end{array}$ & 8.653 & 252 & 218 & 6 & 8 & 8 & 68 \\
\hline & Título & 228 & 3 & 16 & 0 & 2 & 8 & 88 \\
\hline & $\begin{array}{l}\text { Seleccio- } \\
\text { nados }\end{array}$ & 99 & 21 & 2 & 2 & 4 & 3 & 3 \\
\hline
\end{tabular}


La decisión de limitar la búsqueda al período 2003-2014 fue arbitraria. Se utilizaron palabras clave en las bases de datos que contaban con descriptores o vocabulario controlado (Thesauros $\mathrm{MeSH}$ : cervix neoplasm, cancer screening, early detection of cáncer, accessibility, health services); DeCS: cáncer del cuello uterino, prevención del cáncer de cuello uterino, acceso a los servicios de salud, detección precoz del cáncer), y libres: diagnóstico tardío, tratamiento tardío, diagnosis delay, treatment delay.

Los criterios de inclusión de los artículos fueron según tipo de publicación, población estudio, idioma del documento original, tipo de documento (sólo con resumen disponible), año de publicación y área geográfica. Luego de incluir cuarenta documentos 'texto completo' derivados de investigación y revisión, exceptuando los clasificados como literatura gris, editoriales, po- nencias, comunicaciones y artículos de opinión, se procedió a la lectura de los mismos, proceso del cual se excluyeron dieciocho documentos por no estar relacionados con el objetivo de esta revisión o no corresponder con el área geográfica de interés. La información se recolectó mediante un protocolo que contemplaba las siguientes variables: a) Aspectos generales de los artículos: año de publicación y disciplina de la revista (especializada en oncología, salud pública o ciencias médicas), y b) Aspectos metodológicos de los estudios: tipo de estudio realizado: empírico o teórico; tipo de metodología aplicada: cuantitativa, cualitativa o ambas; tipo de diseño de los estudios: de cohorte, de casos y controles, transversales, ecológicos, poblacionales o cualitativos, $\mathrm{y}$ objeto principal del estudio: barreras socioculturales, barreras psicológicas, barreras de sistemas de salud y acceso en Tabla 2.

Tabla 2. Criterios para la selección de los documentos de la revisión sistemática exploratoria

\begin{tabular}{ll}
\hline Introducción & \\
\hline \multirow{2}{*}{ Pregunta de Investigación } & $\begin{array}{l}\text { ¿Cuál es la literatura existente sobre barreras de acceso } \\
\text { detección oportuna del cáncer cervical? }\end{array}$ \\
Objetivo & $\begin{array}{l}\text { Realizar un scoping review o revisión sistemática exploratoria } \\
\text { que responda la pregunta. }\end{array}$
\end{tabular}

Metodología

Periodo de estudio: 2003-2014

Idiomas: inglés y castellano

Tipo de Publicación: especializada en oncología, salud

Criterios de Inclusión pública o ciencias médicas

Población estudio: mujeres con cáncer cervicouterino Área geográfica: Colombia y sus regiones 
Continuación Tabla 2. Criterios para la selección de los documentos de la revisión sistemática exploratoria

\begin{tabular}{|c|c|}
\hline Fuentes de Información & $\begin{array}{l}\text { PubMed, LILACS, The Cochrane Library, Cancer Library, } \\
\text { CANCERMondial, Ministerio de Salud y Protección Social y } \\
\text { repositorios institucionales. }\end{array}$ \\
\hline Estrategia de Búsqueda & $\begin{array}{l}\text { Descriptores Thesauros. MeSH para PubMed. DeCs } \\
\text { para LILACS. Lenguaje libre para todas las fuentes de } \\
\text { información. }\end{array}$ \\
\hline $\begin{array}{l}\text { Selección y Clasificación de } \\
\text { Estudios }\end{array}$ & $\begin{array}{l}\text { 1. Empírico o teórico; } \\
\text { 2. Tipo de metodología aplicada: cuantitativa, cualitativa } \\
\text { o ambas; } \\
\text { 3. Tipo de diseño de los estudios: de cohorte, de casos y } \\
\text { controles, transversales o cualitativos. }\end{array}$ \\
\hline $\begin{array}{l}\text { Extracción de Datos } \\
\text { Resultados }\end{array}$ & Lectura crítica y obtención de información. \\
\hline $\begin{array}{l}\text { Resumen del Número de } \\
\text { Artículos Incluidos en la } \\
\text { Revisión }\end{array}$ & $\begin{array}{l}\text { Incluidos: } 22 \\
\text { Excluidos: } 18\end{array}$ \\
\hline
\end{tabular}

\section{Resultados}

\section{Barreras socioculturales}

Algunas de las razones descritas por las mujeres colombianas para no obtener los resultados de su citología, fueron barreras socioculturales, siendo estas: la no entrega de los resultados por parte de la institución y la falta de interés en el resultado por parte de ellas. Las principales razones para no tener un seguimiento de una citología anormal fueron pereza/falta de interés y falta de recursos económicos. Casi la mitad de las mujeres sin cobertura de salud reportaron falta de recursos económicos como un motivo para no tener un seguimiento de anormalidades citológicas, comparado con una cuarta parte de las mujeres afiliadas al régimen subsidiado $\mathrm{y}$ una novena parte afiliadas al régimen contributivo (10). Asimismo la evidencia afirma que las mujeres jóvenes o mayores tienden a recibir menos seguimiento; contrariamente, las mujeres con más altos ingresos (14) o con seguro de salud privado tienden a participar más del seguimiento $(15,16)$. En Colombia también se reporta la existencia de barreras socioculturales asociadas la edad, el estado civil, el nivel educativo, la procrastinación, necesidades de la familia, sentimientos de: pena, miedo al dolor, miedo de ser diagnosticado con cáncer y fatalismo, así como oposición de la pareja y falta de redes sociales que impiden el oportuno diagnóstico de esta enfermedad (17-21) en Tabla 3.

\section{Barreras psicológicas}

De acuerdo con la reciente Encuesta Na- 
poco menos de la mitad de las mujeres que no se han hecho la citología adujeron sentimientos de miedo o temor, seguido de pereza o descuido (11). Entre los factores psicosociales se mencionan el temor a ser diagnosticada con cáncer, las preocupaciones acerca de la toma de exámenes y/o tratamientos, y la presencia de una red de apoyo social (22). Cabe destacar que las mujeres con pareja o con algún tipo de apoyo social fueron más dadas a tener un seguimiento que aquellas sin apoyo social (23). Un estudio conducido por Nelson et al., encontró que las mujeres que demoraban su seguimiento tenían puntos de vista más fatalistas acerca del cáncer y reportaban más ideas equivocadas acerca del cáncer de cuello uterino (24) en Tabla 3.

\section{Barreras del sistema de salud y de acceso}

Este tipo de barreras de acceso están asociadas a factores estructurales y socio-económicos, por ejemplo Lucumi-Cuesta reportó que las mujeres con cobertura de salud y una fuente regular de atención en salud suelen obtener una citología en los últimos tres años (25). Contrariamente, Piñeros et al en un análisis de la Encuesta de Demografía y Salud reportaron que las mujeres más pobres y con menos educación no suelen obtener una citología (20) en Tabla 3.

Un antecedente importante a mencionar, es que en Colombia los resultados del programa nacional de tamización han sido limitados (26), puesto que presentan bajos indicadores de estructura, proceso y resultado (27) y ahí las actividades de tamización y tratamiento no se articulan (28). Por otro lado, en Colombia la repetición constante y la falta de un seguimiento sistematizado de mujeres con anormalidades citológicas no aseguran un diagnóstico y tratamiento oportuno (27). Por consiguiente la literatura ha demostrado que las tasas de mortalidad en Colombia están relacionadas con las bajas tasas de seguimiento de citologías anormales no con la cobertura del tamizaje como se ha creído $(29,30)$ en Tabla 3.

La ENDS afirma que las mujeres que nunca se han hecho una citología habitan, mayormente, en zonas rurales, en las zonas con menor índice de riqueza y con centros de salud deficientes (10). Tales deficiencias han sido evidenciadas en la falta de seguro de salud, las largas filas y trámites que deben hacer los pacientes para acceder a los servicios, la larga espera por los resultados, la mala atención por parte de los proveedores de salud y a que los proveedores de salud no brindan explicaciones sobre los procedimientos (31). Las largas filas, las numerosas autorizaciones y papeleos, el servicio deficiente y la falta de coordinación acerca de quién provee y paga por ciertos servicios es variable, esto de acuerdo al tipo de cobertura, y a la entidad prestadora del servicio (20)(32-35).

En Colombia, de acuerdo con el Registro Individual de Prestación de Servicios (RIPS), presentado en el Análisis de Situación en Salud 2002-2007, las lesiones más severas NIC II y III predominaron en mujeres del régimen subsidiado y en aquellas sin aseguramiento. Mientras que las acciones para el control y el tratamiento de la enfermedad son inferiores 
en mujeres afrodescendientes, indígenas, palenqueras y raizales pertenecientes a las clases más pobres del país, las cuales carecen de empleo y educación (11) en Tabla 3.

Finalmente, un estudio de Wiesner-Ceballos et al realizado en Boyacá, Caldas,
Magdalena y Tolima con actores del sistema de salud, encontró que el interés de los gestores y prestadores de servicios de salud se ha centrado principalmente en los intereses económicos del sistema y por tanto, no hay un seguimiento adecuado de los casos (36) en Tabla 3.

Tabla 3. Estudios sobre barreras socioculturales, psicológicas y de sistemas de salud y acceso que impiden el diagnóstico oportuno del cáncer cervicouterino en Colombia.

\begin{tabular}{|c|c|c|c|c|c|}
\hline Documento & Autor & Año & $\begin{array}{l}\text { Tipo de } \\
\text { Estudio }\end{array}$ & Barrera & Fuente \\
\hline $\begin{array}{l}\text { Salud sexual y reproductiva en } \\
\text { Colombia. En: Encuesta Nacional } \\
\text { de Demografía y Salud. }\end{array}$ & $\begin{array}{l}\text { Ojeda G, } \\
\text { Ordoñez M, } \\
\text { Ochoa L }\end{array}$ & 2005 & $\begin{array}{l}\text { Encuesta } \\
\text { Poblacional }\end{array}$ & \multirow{5}{*}{ 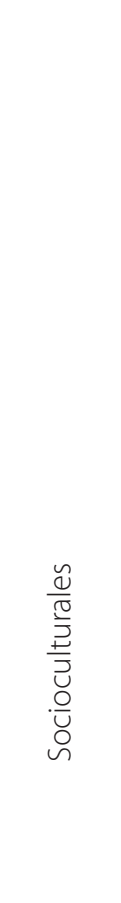 } & $\begin{array}{l}\text { Ministerio } \\
\text { de Salud y } \\
\text { Protección } \\
\text { Social }\end{array}$ \\
\hline $\begin{array}{l}\text { Detección temprana del cáncer } \\
\text { de cuello uterino y de mama. } \\
\text { En: Encuesta Nacional de } \\
\text { Demografía y Salud. }\end{array}$ & $\begin{array}{l}\text { Ojeda G, } \\
\text { Ordoñez M, } \\
\text { Ochoa L }\end{array}$ & 2010 & $\begin{array}{l}\text { Encuesta } \\
\text { Poblacional }\end{array}$ & & $\begin{array}{l}\text { Ministerio } \\
\text { de Salud y } \\
\text { Protección } \\
\text { Social }\end{array}$ \\
\hline $\begin{array}{l}\text { Nuevos paradigmas y desafíos } \\
\text { en la prevención y control del } \\
\text { cáncer de cuello uterino en } \\
\text { América Latina. }\end{array}$ & $\begin{array}{l}\text { Almonte M, } \\
\text { Murillo R, } \\
\text { Sánchez Gl, } \\
\text { Jerónimo J, } \\
\text { Salmerón J }\end{array}$ & 2010 & Revisión & & PubMed \\
\hline $\begin{array}{l}\text { Análisis de situación de salud } \\
\text { según regiones Colombia. }\end{array}$ & $\begin{array}{l}\text { Ministerio } \\
\text { de Salud y } \\
\text { Protección } \\
\text { Social }\end{array}$ & 2013 & Poblacional & & $\begin{array}{l}\text { Ministerio } \\
\text { de Salud y } \\
\text { Protección } \\
\text { Social }\end{array}$ \\
\hline $\begin{array}{l}\text { Cobertura de la citología de } \\
\text { cuello uterino y factores } \\
\text { relacionados en Colombia, } 2005 .\end{array}$ & $\begin{array}{l}\text { Piñeros M, } \\
\text { Cendales R, } \\
\text { Murillo R, } \\
\text { Wiesner C }\end{array}$ & 2007 & Transversal & & PubMed \\
\hline $\begin{array}{l}\text { Factores asociados con el } \\
\text { tamizaje de cáncer de cuello } \\
\text { uterino en mujeres de nivel } \\
\text { socioeconómico medio y bajo } \\
\text { en Bogotá, Colombia. }\end{array}$ & $\begin{array}{l}\text { Garcés I, } \\
\text { Rubio DC, } \\
\text { Scarinci IC }\end{array}$ & 2012 & Cualitativo & & PubMed \\
\hline $\begin{array}{l}\text { La citología de cuello uterino en } \\
\text { Soacha, Colombia: } \\
\text { Representaciones Sociales, } \\
\text { Barreras y Motivaciones. }\end{array}$ & $\begin{array}{l}\text { Wiesner C, } \\
\text { Vejarano- } \\
\text { Velandia M, } \\
\text { Caicedo- } \\
\text { mera JC }\end{array}$ & 2006 & Cualitativo & & PubMed \\
\hline
\end{tabular}


Continuación Tabla 3. Estudios sobre barreras socioculturales, psicológicas y de sistemas de salud y acceso que impiden el diagnóstico oportuno del cáncer cervicouterino en Colombia.

\begin{tabular}{|c|c|c|c|c|c|}
\hline Documento & Autor & Año & $\begin{array}{l}\text { Tipo de } \\
\text { Estudio }\end{array}$ & Barrera & Fuente \\
\hline $\begin{array}{l}\text { Factores socio-cognitivos } \\
\text { asociados a la práctica de la } \\
\text { citología vaginal en mujeres de } \\
\text { nivel socioeconómico medio y } \\
\text { bajo de Bogotá D.C. }\end{array}$ & $\begin{array}{l}\text { Loaiza A, } \\
\text { Gómez S, } \\
\text { Lucumí D }\end{array}$ & 2003 & $\begin{array}{l}\text { Informe } \\
\text { Técnico }\end{array}$ & 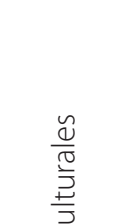 & $\begin{array}{l}\text { Ministerio } \\
\text { de Salud y } \\
\text { Protección } \\
\text { Social }\end{array}$ \\
\hline $\begin{array}{l}\text { Asistencia a citología del cuello } \\
\text { uterino y sus determinantes en } \\
\text { una población rural Colombiana, } \\
\text { 1998-1999. }\end{array}$ & $\begin{array}{l}\text { Castro- } \\
\text { Jiménez M, } \\
\text { Londoño- } \\
\text { Cuellar P, } \\
\text { Vera-Cala L }\end{array}$ & 2006 & Transversal & $\begin{array}{l}\text { Ð } \\
\text { Oั } \\
\text { O }\end{array}$ & PubMed \\
\hline $\begin{array}{l}\text { Detección temprana del cáncer } \\
\text { de cuello uterino y de mama. } \\
\text { En: Encuesta Nacional de } \\
\text { Demografía y Salud. }\end{array}$ & $\begin{array}{l}\text { Ojeda G, } \\
\text { Ordoñez M, } \\
\text { Ochoa L }\end{array}$ & 2010 & $\begin{array}{l}\text { Encuesta } \\
\text { Poblacional }\end{array}$ & $\begin{array}{l}\frac{n}{0} \\
\frac{0}{8} \\
\frac{0}{0} \\
\frac{u}{n} \\
\curvearrowleft\end{array}$ & $\begin{array}{l}\text { Ministerio } \\
\text { de Salud y } \\
\text { Protección } \\
\text { Social }\end{array}$ \\
\hline $\begin{array}{l}\text { Salud sexual y reproductiva en } \\
\text { Colombia. En: Encuesta Nacional } \\
\text { de Demografía y Salud. }\end{array}$ & $\begin{array}{l}\text { Ojeda G, } \\
\text { Ordoñez M, } \\
\text { Ochoa L }\end{array}$ & 2005 & $\begin{array}{l}\text { Encuesta } \\
\text { Poblacional }\end{array}$ & & $\begin{array}{l}\text { Ministerio } \\
\text { de Salud y } \\
\text { Protección } \\
\text { Social }\end{array}$ \\
\hline $\begin{array}{l}\text { Accessibility to healthcare } \\
\text { services in the recent cervical } \\
\text { cytology performed in an urban } \\
\text { area in Colombia. }\end{array}$ & $\begin{array}{l}\text { Lucumí } \\
\text { Cuesta D, } \\
\text { Gómez- } \\
\text { Gutiérrez L }\end{array}$ & 2004 & Transversal & & PubMed \\
\hline $\begin{array}{l}\text { Efectividad de la citología } \\
\text { cérvico-uterina para la } \\
\text { detección temprana de cáncer } \\
\text { de cuello uterino en el marco } \\
\text { del sistema de salud de } \\
\text { Colombia. }\end{array}$ & $\begin{array}{l}\text { Murillo R, } \\
\text { Cendales R, } \\
\text { Wiesner C, } \\
\text { Piñeros M, } \\
\text { Tovar S }\end{array}$ & 2009 & $\begin{array}{l}\text { Casos y } \\
\text { Controles }\end{array}$ & 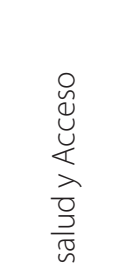 & PubMed \\
\hline $\begin{array}{l}\text { Nuevos paradigmas y desafíos } \\
\text { en la prevención y control del } \\
\text { cáncer de cuello uterino en } \\
\text { América Latina. }\end{array}$ & $\begin{array}{l}\text { Almonte M, } \\
\text { Murillo R, } \\
\text { Sánchez Gl, } \\
\text { Jerónimo J, } \\
\text { Salmerón J }\end{array}$ & 2010 & Revisión & $\begin{array}{l}0 \\
0 \\
0 \\
\tilde{0} \\
\frac{\varepsilon}{4} \\
\stackrel{\oplus}{n}\end{array}$ & PubMed \\
\hline $\begin{array}{l}\text { Seguimiento de mujeres con } \\
\text { anormalidad citológica de } \\
\text { cuello uterino en Colombia. }\end{array}$ & $\begin{array}{l}\text { Wiesner } C, \\
\text { Cendales R, } \\
\text { Murillo R, } \\
\text { Piñeros M }\end{array}$ & 2010 & Transversal & & PubMed \\
\hline $\begin{array}{l}\text { Cost-effectiveness of } \\
\text { conventional cytology and HPV } \\
\text { DNA testing for cervical cancer } \\
\text { screening in Colombia. }\end{array}$ & $\begin{array}{l}\text { Gamboa A, } \\
\text { Chicaíza L, } \\
\text { García- } \\
\text { Molina M, } \\
\text { et al }\end{array}$ & 2008 & $\begin{array}{l}\text { Análisis de } \\
\text { Costo } \\
\text { Efectividad }\end{array}$ & & PubMed \\
\hline
\end{tabular}


Continuación Tabla 3. Estudios sobre barreras socioculturales, psicológicas y de sistemas de salud y acceso que impiden el diagnóstico oportuno del cáncer cervicouterino en Colombia.

\begin{tabular}{|c|c|c|c|c|c|}
\hline Documento & Autor & Año & $\begin{array}{l}\text { Tipo de } \\
\text { Estudio }\end{array}$ & Barrera & Fuente \\
\hline $\begin{array}{l}\text { How protective is cervical } \\
\text { cancer screening against cervi- } \\
\text { cal cancer mortality in develo- } \\
\text { ping countries? The Colombian } \\
\text { case. }\end{array}$ & $\begin{array}{l}\text { Chocontá- } \\
\text { Piraquive LA, } \\
\text { Alvis- } \\
\text { Guzmán N, } \\
\text { Hoz- } \\
\text { Restrepo F } \\
\text { De }\end{array}$ & 2010 & Ecológico & & PubMed \\
\hline $\begin{array}{l}\text { Factores asociados a la } \\
\text { práctica de la citología de cuello } \\
\text { uterino, en mujeres desplazadas } \\
\text { y población receptora en un } \\
\text { asentamiento en Antioquia, } \\
\text { Colombia, } 2011 .\end{array}$ & $\begin{array}{l}\text { Ramos S, } \\
\text { Garcés I }\end{array}$ & 2011 & Transversal & & LILACS \\
\hline $\begin{array}{l}\text { Impact of health care coverage } \\
\text { and other socio-demographic } \\
\text { variables on the follow-up } \\
\text { of cervical cancer screening } \\
\text { among Colombian women. }\end{array}$ & Garcés I. & 2009 & Transversal & 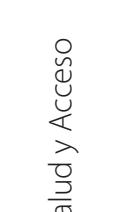 & Repositorio \\
\hline $\begin{array}{l}\text { Conocimiento, actitudes y } \\
\text { prácticas de la citología cérvico } \\
\text { uterina, en mujeres mayores de } \\
15 \text { años en condición de despla- } \\
\text { zamiento, en el barrio Las Flores; } \\
\text { sector Caguan, Chipi y Tambos; } \\
\text { del Distrito de } \\
\text { Barranquilla. } 2010 .\end{array}$ & $\begin{array}{l}\text { Figueroa } \\
\text { Solano, NM }\end{array}$ & 2011 & Transversal & 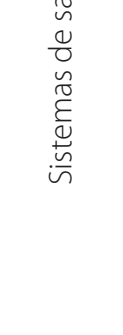 & Repositorio \\
\hline $\begin{array}{l}\text { La otra cara del cáncer } \\
\text { cervicouterino: Un análisis } \\
\text { desde la clase social y la cultura } \\
\text { de género. }\end{array}$ & $\begin{array}{l}\text { Valencia } \\
\text { Arredondo } \\
\text { M, Estrada JH }\end{array}$ & 2013 & Cualitativo & & Repositorio \\
\hline $\begin{array}{l}\text { Control del cáncer } \\
\text { cervicouterino en Colombia : la } \\
\text { perspectiva de los actores del } \\
\text { sistema de salud. }\end{array}$ & $\begin{array}{l}\text { Wiesner C, } \\
\text { Murillo R, } \\
\text { Piñeros M, } \\
\text { Tovar-Murillo } \\
\text { S, Cendales R, } \\
\text { Gutiérrez M. }\end{array}$ & 2009 & Cualitativo & & PubMed \\
\hline
\end{tabular}

\section{Discusión}

Esta revisión ha revelado que la morbilidad y la mortalidad en el país no se distribuyen de manera similar en los territorios. Lo anterior puede ser corroborado si se revisan los reportes de cinco registros poblacionales de cáncer 
geografía colombiana, tales como: Cali, Bucaramanga, Barranquilla y Manizales (37) y Pasto (38). Igualmente hallazgos en Antioquia entre 2000-2007, confirman que las subregiones más alejadas y con más necesidades básicas pueden incluso duplicar la tasa de mortalidad nacional (39). El Atlas de mortalidad por cáncer en Colombia, muestra que la oportunidad en el diagnóstico de cáncer manifestado en la morbi-mortalidad es mayor en las zonas aledañas a los ríos navegables y las áreas de frontera (40), como por ejemplo sucede con la costa Pacífica (41) y en el departamento de Nariño (42).

Indudablemente la revisión confirma hallazgos que indican que los programas de detección temprana del $\mathrm{CaCu}$ en poblaciones mayoritariamente rurales y constituidas por minorías étnicas, son un desafío para Colombia ya que en departamentos fronterizos con altos niveles de población indígena, como La Guajira y el Amazonas, se presentan porcentajes de tamización anual de $37.9 \%$ y $49.7 \%$ respectivamente (10). Se deduce además, que los programas de detección existentes no fueron parte de una fase de consenso con la comunidad, tal como lo demanda el Plan Decenal de Salud Pública 2012-2021, puesto que el cáncer cervico-uterino es una prioridad en salud pública (43). Aunado a esto las investigaciones sobre barreras de acceso en el país siguen siendo escasas y casi nulas en regiones fronterizas y con un alto porcentaje de minorías étnicas.

Hay amplias diferencias económicas y sociales en la situación de salud entre las regiones de Colombia (10), y esto no es la excepción para el cáncer cervico-uterino (13). Las diferencias en situación de salud van de la mano con las diferencias en indicadores económicos y sociales. La incidencia de pobreza en Colombia para el año 2011 fue de 34,1\% y el índice de Gini de 0,548, mostrando ser un país muy desigual; por ejemplo los departamentos con mayor desigualdad para el año 2011 estuvo La Guajira, cuyos índices oscilaron entre 0,554 y 0,567 , mientras en la región Central departamentos como Antioquia y la región Bogotá-Cundinamarca ha disminuido la desigualdad de ingreso (44).

Las barreras de acceso son evidentes, ya que Colombia ha experimentado un descenso progresivo en las tasas de mortalidad, más profundo en los centros urbanos y regiones desarrolladas, mientras que las regiones de menor desarrollo social y económico, la mortalidad tiende a aumentar o permanecer estable como sucede en departamentos como Nariño que en 1990-1996 era de 9.4 y asciendió a 11.3 en 2000-2006 (40).

La situación del cáncer cervico-uterino mostrado en esta revisión, revela similitud con hallazgos en el mundo, donde también el riesgo de cáncer cervical es mayor en comunidades en desventaja, que habitan zonas rurales, zonas de frontera y de bajo recursos, con dificultades de movilidad y falta de transporte (45). La distribución diferencial de la mortalidad del $\mathrm{CaCu}$ en países de Latinoamérica refleja desigualdades socioeconómicas frente al riesgo de morir de las mujeres según grupos sociales $y$ 
regiones. El desarrollo de los programas es diferente según las características de cada región y dentro de los mismos países y para la evaluación del impacto del programa de tamización se utilizan indicadores de morbilidad que sólo cuantifican la reducción en la incidencia de las formas invasivas, y la medición de la mortalidad (46). En Colombia es difícil hacer esa comparación, no sólo por la limitación de evidencia científica sino también por las limitaciones de información en las regiones como la Orinoquia y Amazonía donde solo se ha obtenido información de las mujeres con residencia en la cabecera, y de incluso Bogotá en zonas marginadas donde converge población desplazada. Lo anterior, también imposibilita una comparación epidemiológica urbano-rural y futuros estudios de acceso a servicios de salud entre ambas zonas (47).

Por último, es importante realizar en Colombia más estudios que arrojen evidencia sobre las diferentes barreras de acceso con diferentes modelos teóricos, ya que si bien hay evidencia de que la falta de práctica de la citología está asociada con el cáncer invasor, no se ha logrado explicar suficientemente, las razones por las cuales en Colombia hay mujeres que no se realizan la prueba, estando disponible la tamización en el primer nivel de atención de forma gratuita. Asimismo, existe desconocimiento sobre la dinámica sociocultural, la adherencia y el costo-efectividad de estos programas de tamizaje (28) en Tabla 3.

La información en cuanto a barreras del seguimiento de anormalidades citológicas en Latinoamérica y especí- ficamente para Colombia es limitada. La información publicada se encuentra principalmente en estudios Anglosajones. Sin embargo, esta información concuerda con algunas de las barreras reportadas para la tamización en Colombia, por lo tanto podría pensarse que factores similares a los experimentado por mujeres de otros países también afectan el seguimiento de anormalidades citológicas en Colombia. Por ejemplo, un artículo de revisión sistemática publicado en el 2007 muestra algunas de estas barreras, entre las que se encuentran las características de las mujeres, factores psicosociales y las características del sistema de salud. Sin embargo, Peterson et al encontraron que las mujeres con seguro de salud público tienden a participar más de un seguimiento que las mujeres con seguro privado (16). De otra parte, mujeres con lesiones menos severas fueron menos propensas a seguir las recomendaciones de seguimiento (48-50).

Finalmente, algunas características del sistema de salud, comprenden la comunicación entre los proveedores de salud y los pacientes, los lugares de atención y la especialidad de los proveedores de salud (51). La comunicación entre proveedores y pacientes acerca de las opciones de seguimiento mostraron estar asociadas con un mayor seguimiento (52-53). Así mismo, se reportó que las clínicas que prestaban el servicio de colposcopia en el mismo sitio tenían tasas de seguimiento más altas (52). Otro estudio mostró que no había diferencias en el seguimiento de pacientes que acudían a servicios de atención público o privado (54). 
En cuanto a las limitaciones de esta revisión no se evaluó la calidad de los estudios encontrados, puesto que no se valoró la heterogeneidad de los estudios revisados y su naturaleza descriptiva no permite realizar asociaciones ni meta-análisis de los resultados. Como fortaleza, se destaca la búsqueda exhaustiva de evidencia en el país nunca antes revisadas sistemáticamente. Esta información es muy útil para que investigadores y grupos se interesen por estudiar las causas y condiciones del diagnóstico tardío del cáncer cervico-uterino en mujeres, relacionadas

\section{Referencias}

1. Ferlay J, Shin H-R, Forman D, Mathers C, Maxwell Parkin D. Estimates of worldwide burden of cancer in 2008: Int J Cancer. 2008;127:2893-2917.

2. John R, Ross H. The Global Economic Cost of Cancer. American C. American Cancer Society, ed. Atlanta, USA: Livestrong; 2010. Pp. 6-17

3. Ferlay J, Soerjomataram I, Dikshit R, Eser S, Mathers C, Rebelo M, Parkin DM, Forman D, Bray F. Cancer incidence and mortality worldwide: sources, methods and major patterns in GLOBOCAN 2012. Int J Cancer. 2015 Mar 1;136(5):E35986.

4. Pan American Health Organisation. Regional Strategy and Plan of Action for Cervical Canceer Prevention and Con- con las barreras de acceso a los servicios de salud, preferiblemente en poblaciones vulnerables.

\section{Agradecimientos}

Al programa de Doctorado en Epidemiología de la Facultad Nacional de Salud Pública de la Universidad de Antioquia.

\section{Conflicto de interés}

No existe conflicto de interés. trol. Washington D.C., USA: Pahowho; 2008:1-4.

5. Luciani S, Cabanes A, Prieto-Lara E, Gawryszewski V. Cervical and female breast cancers in the Americas : current situation and opportunities for action. Bull World Heal Organ. 2013;91:640649.

6. Yang BH, Bray FI, Parkin DM, Sellors JW, Zhang Z-F. Cervical cancer as a priority for prevention in different world regions: an evaluation using years of life lost. Int J Cancer. 2004;109(3):418424.

7. Goldie SJ, Gaffikin L, Goldhaber-Fiebert JD, et al. Cost-effectiveness of cervical-cancer screening in five developing countries. N Engl J Med. 2005;353(20):2158-2168. 
8. Insinga RP, Dasbach EJ, Elbasha EH. Assessing the annual economic burden of preventing and treating anogenital human papillomavirus-related disease in the US: analytic framework and review of the literature. Pharmacoeconomics. 2005;23(11):1107-22

9. Bray $F$, Loos $A H$, Mccarron $P$, et al. Trends in Cervical Squamous Cell Carcinoma Incidence in 13 European Countries: Changing Risk and the Effects of Screening Trends in Cervical Squamous Cell Carcinoma Incidence in 13 European Countries : changing Risk and the Effects of Screening. Cancer Epidemiol Biomarkers Prev 2005;14677-686. 2005;14:677686.

10. Ojeda G, Ordoñez M, Ochoa L. Salud Sexual y Reproductiva en Colombia. En: encuesta nacional de demografia y salud. ; 2005:383-409.

11. Ojeda G, Ordoñez M, Ochoa L. Detección temprana del cáncer de cuello uterino y de mama. En: encuesta nacional de demografia y salud. 2010;439-475.

12. Almonte M, Murillo R, Sánchez GI, Jerónimo J, Salmerón J. Nuevos paradigmas y desafíos en la prevención y control del cáncer de cuello uterino en América Latina. Salud Publica Mex. 2010;52(6):544559.

13. Ministerio de Salud y Protección Social. Análisis de Situación de Salud Según Regiones Colombia. Ministerio [Internet]. Grupo ASIS D de E y DM de S y PS, ed. Bogotá, Colombia; 2013. Consultado el 21 de enero de 2015, citado 23 de mayo de 2015. Disponible en: https://www.minsalud.gov.co/Documentos $\% 20 \mathrm{y} \% 20 \mathrm{Pu}$ blicaciones/An\%C3\%A1lisis\%20de \%20 situaci $\%$ C3\%B3n $\% 20$ de $\% 20$ salud $\% 20$ por\%20regiones.pdf

14. Hartz L, Fenaughty A. Management choice and adherence to follow-up after colposcopy in women with cervical intraepithelial neoplasia 1. Obs Gynecol. 2001;98(4):674-679.
15. Wang $H$, Dwyer-lindgren L, Lofgren $\mathrm{KT}$, et al. Age-specifi $\mathrm{c}$ and sex-specifi $\mathrm{C}$ mortality in 187 countries , $1970-2010$ : a systematic analysis for the Global Burden of Disease Study 2010. 2012:4-15.

16. Peterson N, Han J, Freund K. Inadequate follow-up for abnormal pap smear in a urban population. J Natl Med Assoc. 2003;95(9):825-832.

17. Garcés I, Rubio DC, Scarinci IC. Factores asociados con el tamizaje de cáncer de cuello uterino en mujeres de nivel socioeconómico medio y bajo en Bogotá, Colombia. Rev Fac Nac Salud Pública. 2012;30(1):7-16.

18. Wiesner C, Vejarano-velandia M, Caicedo-mera JC. La Citología de Cuello Uterino en Soacha, Colombia: Representaciones Sociales, Barreras y Motivaciones. Rev Salud Publica. 2006;8(3):185-196.

19. Loaiza A, Gómez S, Lucumí D. Factores socio-cognitivos asociados a la práctica de la citología vaginal en mujeres de nivel socioeconómico medio y bajo de Bogotá D.C. Estudio Exploratorio. Bogotá, DC; 2003.

20. Piñeros $M$, Cendales $R$, Murillo $R$, Wiesner C. Cobertura de la Citología de Cuello Uterino y Factores Relacionados en Colombia, 2005. Rev salud pública. 2007;9(3):327-341.

21. Castro-Jiménez M, Londoño-Cuellar P, Vera-Cala L. Asistencia a citología del cuello uterino y sus determinantes en una población rural colombiana, 19981999. Rev salud pública. 2006;8(3):248257.

22. Gómez E. Equidad, género y salud: retos para la acción. Rev Panam Salud Publica/Pan Am J Public Heal. 2002;11(5/6):454-461.

23. Woods LM, Rachet B, Coleman MP. Origins of socio-economic inequalities in cancer survival: a review. Ann Oncol. 
24. Nelson K, Geiger AM, Mangione $\mathrm{CM}$. Effect of health beliefs on delays in care abnormal cervical cytology in a multiethnic population. J Gen Intern Med. 2002;17:709-716.

25. Lucumí Cuesta D, Gómez-Gutiérrez L. Accessibility to healthcare services in the recent cervical cytology performed in an urban area in Colombia. Rev salud pública. $2004 ; 78(3): 367-377$.

26. Almonte M, Murillo R, Sánchez GI, Jerónimo J, Salmerón J. Nuevos paradigmas y desafíos en la prevención y control del cáncer de cuello uterino en América Latina. Salud Publica Mex. 2010;52(6):544559.

27. Wiesner C, Cendales R, Murillo R, Piñeros $M$. Seguimiento de mujeres con anormalidad citológica de cuello uterino, en Colombia. Rev Salud Publica. 2010;12(1):1-13.

28. Murillo R, Cendales R, Wiesner $\mathrm{C}$, Piñeros M, Tovar S. Efectividad de la citología cérvico-uterina para la detección temprana de cáncer de cuello uterino en el marco del sistema de salud de Colombia. Biomédica. 2009;29:354-361.

29. Gamboa A, Chicaíza L, García-Molina $M$, et al. Cost-effectiveness of conventional cytology and HPV DNA testing for cervical cancer screening in Colombia. Salud Publica Mex. 2008;50(9):276-285.

30. Chocontá-piraquive LA, Alvis-guzman $\mathrm{N}$, Hoz-restrepo F De. How protective is cervical cancer screening against cervical cancer mortality in developing countries ? The Colombian case. 2010:2-7.

31. Ramos S, Garcés I. Factores asociados a la práctica de la citología de cuello uterino, en mujeres desplazadas y población receptora en un asentamiento en Antioquia , Colombia , 2011. Rev Fac Nac Salud Pública. 2013;31(3):311-318.

32. Pinilla J. Bogotá's Administrative Health Department, Bogotá, Colombia.
Personal Communication. October 10, 2007.

33. Figueroa Solano NM. Conocimiento, actitudes y prácticas de la citología cervico uterina, en mujeres mayores de 15 años en condición de desplazamiento, en el barrio Las Flores; sector Caguan, Chipi y Tambos; del Distrito de Barranquilla. 2010. 2011.

34. Garcés IC, Altarac M, Kirby DR, Mcclure LA, Mulvihill B, Scarinci IC. Contribution of Health Care Coverage in Cervical Cancer Screening Follow-Up Findings From a Cross-Sectional Study in Colombia. Int J Gynecol Cáncer. 2010; 20(7):1232-1239.

35. Valencia-Arredondo M. Otra Cara del Cáncer Cervicouterino: una mirada desde la clase social y la cultura de género [Investigación]. Medellín: Universidad de Antioquia; 2013. Pp. 27-54.

36. Wiesner $C$, Murillo R, Piñeros M, Tovar-Murillo $S$, Cendales R, Gutiérrez M. Control del cáncer cervicouterino en Colombia : la perspectiva de los actores del sistema de salud. Rev Panam Salud Pública. 2009;25(1):1-8.

37. Muñoz N, Bravo Ocaña LE. Epidemiology of cervical cancer in Colombia. Colomb Med. 2012;43(4):298-304.

38. Yepez, M., Bravo, L., Troya, A., Jurado, D., \& Bravo, L. 2012 Dec 29. Cancer incidence and mortality in the municipality of Pasto, 1998 - 2007. Colombia Médica. 2012; 43:4: 256-6.

39. Baena A, Almonte M, Valencia ML, et al. Tendencias e indicadores sociales de la mortalidad por cáncer de mama y cueIlo. Salud Publica Mex. 2011;53(6):486492.

40. Piñeros Petersen $M$, Pardo Ramos C, Gamboa Garay Ó, Hernández Suárez G. Atlas de mortalidad por cáncer en Colombia. Instituto Nacional de Cancerología, Ministerio Protección Social, Instituto 
Geográfico Agustín Codazzi, eds. Bogotá, Colombia; 2010. Pp. 23

41. Rocha-Buelvas A, Trujillo-Montalvo E, Hidalgo-Patiño C, Hidalgo-Eraso Á. Disease burden in Nariño, Colombia, 2010. Colomb Med. 2014;45(3):54-61.

42. Rocha-Buelvas A, Trujillo-Montalvo $E$, Hidalgo-Patiño $C$, Hidalgo-Eraso Á. Carga de cáncer del departamento de Nariño y subregiones, Colombia, 2010. Rev Fac Nac Salud Publica. 2014;32(3):340-354.

43. Ministerio de Salud y Protección Social, Instituto Nacional de Cancerología. Plan Decenal Para El Control Del Cáncer En Colombia 2012-2021. (Ministerio de Salud y Protección Social, Instituto Nacional de Cancerología, eds.). Bogotá, Colombia; 2012.

44. Ministerio de Salud y Protección Social. Análisis de Situación de Salud Según Regiones Colombia. Bogotá, Colombia: Ministerio de Salud y Protección Social; 2013.

45. Parikh S, Brennan P, Boffetta P. Meta-analysis of social inequality and the risk of cervical cancer. Int $\mathrm{J}$ Cancer. 2003;105:687-691.

46. Lazcano-Ponce E, Alonso P, Ruiz-Moreno JA, Hernández-Avila $M$. Recommendations for cervical cancer screening programs in developing countries: the need for equity and technological development.Salud pública Méx. 2003; 45 (3): 449-462.

47. Facultad Nacional de Salud Publica $\mathrm{U}$ de A. Tomo II. Salud sexual y repro- ductiva. Análisis de la situación de salud en Colombia, 2002-2007.; 2010. Pp. 5274.

48. Melnikow J, Chan B, Stewart G. Do follow-up recommendations for abnormal Papanicolaou smears influence patient adherence? Arch Fam Med. 1999;8(6):510-514.

49. Fox $P$, Amsberger $P$, Zhang $X$. An examination of differential follow-up rates in cervical cancer screening. J Community Heal. 1997;22(3):199-209.

50. Benard V, HW L, Eheman C, Anderson $\mathrm{C}$, Helsel W. Adherence to guidelines for follow-up of low-grade cytologic abnormalities among medically underserved women. Obs Gynecol. 2005;105(6):13231328.

51. Eggleston K, Coker A, Prabhu I, Cordray S, Luchok K. Understanding Barriers for Adherence to Follow-Up Care for Abnormal Pap Tests. J Women's Heal. 2007;16(3):311-330.

52. McKee M, Lurio J, P M, Burton W, M M. Barriers to Follow-up of Abnormal Papanicolaou Smears in an Urban Community Health Center. Arch Fam Med. 1999;8:129-134.

53. McKee M, Schechter C, Burton W, Mulvihill M. Predictors of follow-up of atypical and ASCUS papanicolaou tests in a high-risk population. J Fam Pr. 2001;50(7):609.

54. Lacey L, Whitfield J, DeWhite W, et al. Referral adherence in an inner city breast and cervical cancer screening program. Cancer. 1993;72(3):950-955 
INVESTIGACIONES ANDINA No. 33 Vol. 18 